\title{
ADVANCED HOMOGENIZATION METHODS FOR PRESSURIZED WATER REACTORS
}

\author{
PAVEL SUK

\begin{abstract}
Department of Nuclear Reactors, Faculty of Nuclear Science and Physical Engineering, Czech Technical
\end{abstract} \\ University in Prague, V Holešovičkách 2, 18000 Prague 8, Czech Republic \\ correspondence: sukpave2@fjfi.cvut.cz
}

\begin{abstract}
Macroscopic cross section generation is key part of core calculation. Commonly, the data are prepared independently without a knowledge of fuel loading pattern. The fuel assemblies are simulated in infinite lattice (with mirror boundary conditions). Rehomogenization method is based on combination of actual neutron flux in fuel assembly with macroscopic data from infinite lattice. Rehomogenization method was implemented into the macrocode Andrea and tested on a reference cases. Cases consist of fuel cases, cases with strong absorber, cases with absorption rods, or cases with reflector assemblies. Testing method is based on a comparisons of homogenized and rehomogenized macroscopic cross sections and later on a comparisons of relative power of each fuel assembly. Above that there is comparison of eigenvalue.
\end{abstract}

KEYWORDS: homogenization, rehomogenization, full core calculation, Andrea, Helios, macroscopic data preparation, neutron flux.

\section{INTRODUCTION AND MAIN MOTIVATION}

Calculation of main neutronic data for power nuclear reactors is complicated multi-level process. Despite steadily rising computational power of computers, there is no way to calculate real fuel loading pattern using accurate deterministic or stochastic codes. This problematic is divided in two levels: preparation of macroscopic data and calculation real core with macrocodes with diffusion or simplified transport solution.

The data preparation process for fuel assemblies is provided by deterministic or stochastic codes (microcodes). Deterministic codes solve transport equation (the SCALE Newt [1, the Helios [2]) and the stochastic codes (the Serpent [3]) simulate the batch of particles. The data for macrocodes are prepared with simulation of identical fuel assemblies in infinite lattice. This approach is called Standard Homogenization Method. The method considered some simplification which are not fulfilled in the real fuel loading pattern. The simplifications are:

- zero neutron escape over the boundary of fuel assembly,

- symmetrical distribution of neutron flux inside fuel assembly,

- energy spectrum of neutron during data preparation process is very different from real energy spectrum.

Comparison of neutron flux inside fuel assembly is illustrated in figure 1. Fuel assembly is simulated in infinite lattice (left) and next to the strong absorber (right). The figure clearly shows that in simulated fuel assembly next to the strong absorber is significant neutron flux tilt, which can't be considered by
Standard Homogenization Method.

The solution of unfulfilled assumptions (symetrical distribution of neutron flux inside fuel assembly and zero escape over the boundary of fuel assembly) of standard data preparation process can be found with using rehomogenization method. The rehomogenization method is based on method cited in article of Aldo Dall'Osso [4, who tested advanced spatial rehomogenization. This method is based on a actual distribution of neutron flux inside fuel assembly during the full core calculation. The data prepared by this method should better reflect spatial dependence of neutron flux during the data preparation process and the neutron escape from fuel assembly.

Since the rehomogenization method depend on fuel pattern, it must be implemented in macrocode, where are the data continuously edited with respect to calculation of main neutron-physical characteristics.

\section{REHOMOGENIZATION THEORY}

The rehomogenization method can be divided into two parts. In the first part, the neutron flux in the fuel assembly is calculated. In the second part, the macroscopic data are prepared with actual neutron flux. Since the main intention of the method is to ensure more realistic data in macrocode, there is need to use data provided by macrocode itself during the calculation.

The interface and mean neutron flux values were used for the neutron flux distribution calculation inside fuel assembly, because the macrocode Andrea calculate them. Neutron fluxes on the interfaces of fuel assembly (interfaces E, NE, NW, W, SW, SE) and mean value of neutron flux in fuel assembly were 


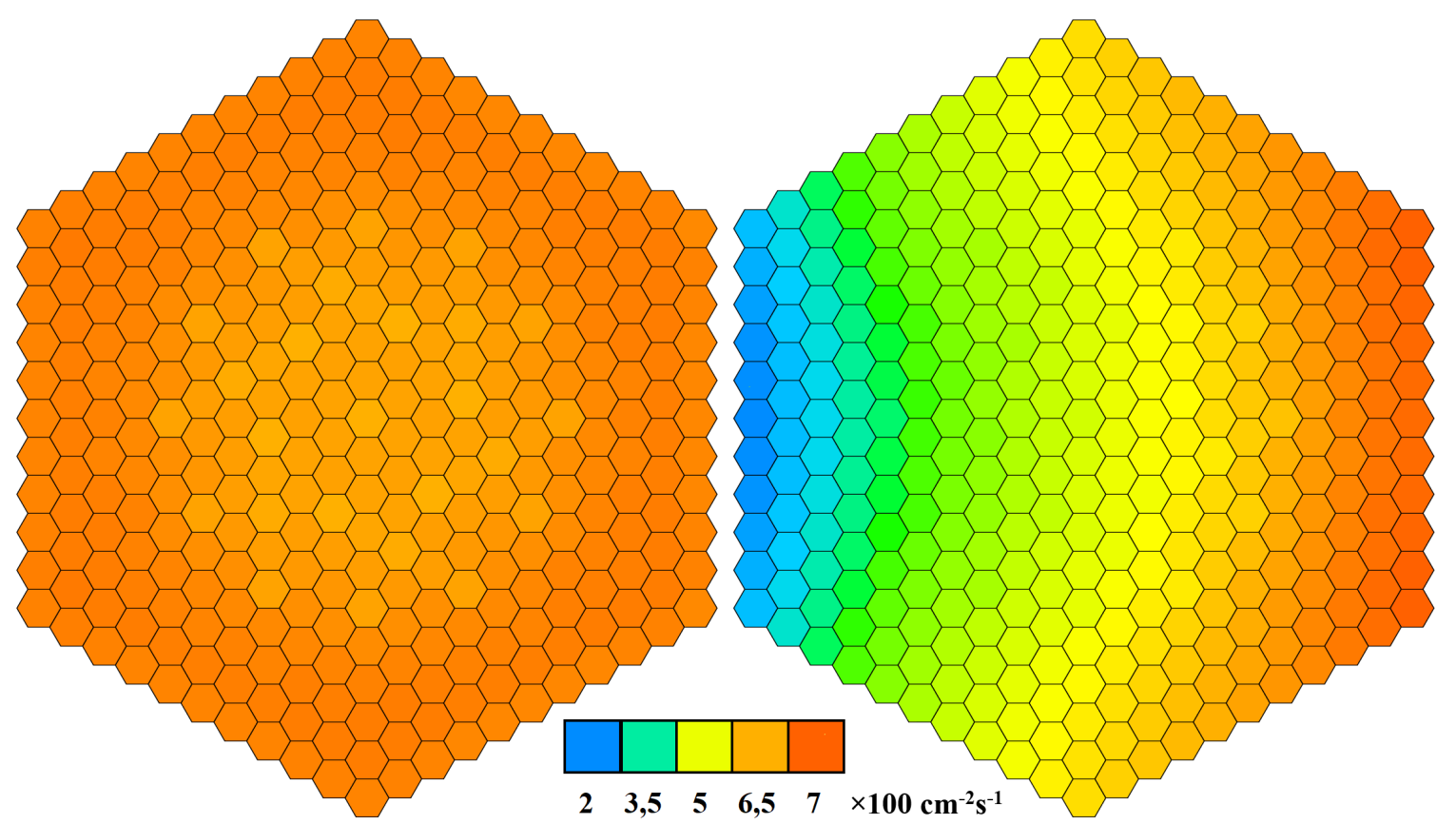

Figure 1. Distribution of neutron flux in fuel assembly simulated in infinite lattice (left) and behind strong absorber (right).

used as input data for calculation of neutron flux distribution.

\subsection{INTERPOLATION OF NEUTRON FLUX}

Calculation of the neutron flux distribution can be done by two dimension interpolation function. In terms of the simple approach of interpolation, the deviation of the neutron flux was interpolated using the following polynomial functions:

$$
\begin{aligned}
\Phi(x, y) & -\bar{\Phi}=C_{1} \cdot x+C_{2} \cdot y+ \\
& +C_{3} \cdot\left(x^{2}+y^{2}-C\right)+C_{4} \cdot\left(x^{2}-y^{2}\right)+ \\
& +C_{5} \cdot x \cdot y .
\end{aligned}
$$

Constants $C_{1}$ to $C_{5}$ are interpolation constants, constant $C$ is orthogonalization constant. To consider local deformation in neutron flux (for example by cell without fuel), the interpolated neutron flux for each cell with coordinates $\left(x_{\mathrm{b}}, y_{\mathrm{b}}\right)$ were multiplied by fac-

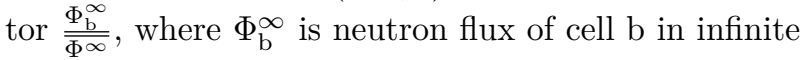
lattice and $\overline{\Phi^{\infty}}$ is mean value of neutron flux in fuel assembly simulated in infinite lattice.

Comparison of interpolated neutron flux with reference values obtained by simulation of case in deterministic code shows that neutron flux cell data does not agree with real distribution of neutron flux in fuel assembly. It turns out that problem is in complete solution of diffusion equation. Interpolation using polynomial function can express only particular solution of diffusion equation, but it can not approximate homogeneous part of solution.

\subsection{ReCOnstruction OF NEUTRON FLUX}

The reconstruction method of neutron flux is more complicated approach in comparison with the interpo- lation method. The reconstruction is based on solution of two groups diffusion equation in fuel assembly:

$$
\nabla^{2} \vec{\Phi}=\left(\begin{array}{cc}
\frac{\Sigma_{\mathrm{a} 1}-\nu \Sigma_{\mathrm{f} 1} / k_{\mathrm{ef}}}{D_{1}} & -\frac{\nu \Sigma_{\mathrm{f} 2} / k_{\mathrm{ef}}+\Sigma_{\mathrm{g} 2 \mathrm{~g} 1}}{D_{1}} \\
-\frac{\Sigma_{\mathrm{g} 1 \mathrm{~g} 2}}{D_{2}} & \frac{\Sigma_{\mathrm{a} 2}}{D_{2}}
\end{array}\right) \vec{\Phi}
$$

where the quantities in equation are:

- $\Sigma_{\mathrm{a} 1}, \Sigma_{\mathrm{a} 2}$ macroscopic cross sections for neutron absorption in energetic groups 1 and 2,

- $\nu \Sigma_{\mathrm{f} 1}, \nu \Sigma_{\mathrm{f} 2}$ macroscopic cross sections for fission in both groups multiplied by average number of neutron from fission,

- $D_{1}, D_{2}$ diffusion coefficient,

- $\Sigma_{\mathrm{g} 1 \mathrm{~g} 2}, \Sigma_{\mathrm{g} 2 \mathrm{~g} 1}$ scattering cross section from first energy group to second and conversely,

- $\vec{\Phi}$ is vector of scalar neutron flux in both groups,

Multiplied equation 2 by vector $\vec{v}$, the diffusion equation can be rewritten to form:

$$
\vec{v} \cdot \nabla^{2} \vec{\Phi}=\vec{v} \cdot \mathbb{M} \vec{\Phi}=\mathbb{M}^{T}(\vec{v} \vec{\Phi})
$$

If is vector $\vec{v}$ chosen as an eigenvector of matrix $\mathbb{M}^{T}$, eigen to eigenvalue $\lambda_{\mathrm{g}}$, for its component applies:

$$
v_{\mathrm{g}}=\left(\mathbb{M}_{21}, \mathbb{M}_{11}-\lambda_{\mathrm{g}}\right)
$$

Equation 3 can be rewritten as two simple wave equations:

$$
\nabla^{2} \xi_{\mathrm{g}}-\lambda_{\mathrm{g}} \xi_{\mathrm{g}}=0,
$$

where $\mathrm{g}$ takes value 1 and 2 . Using vector form, the system can be written as:

$$
\vec{\xi}=\mathbb{R} \vec{\Phi},
$$


where matrix $\mathbb{R}$ is:

$$
\mathbb{R}=\left(\begin{array}{cc}
\frac{\Sigma_{\mathrm{a} 1}-\nu \Sigma_{\mathrm{f} 1} / k_{\mathrm{ef}}}{D_{1}} & -\frac{\nu \Sigma_{\mathrm{f} 2} / k_{\mathrm{ef}}+\Sigma_{\mathrm{g} 2 \mathrm{~g} 1}}{D_{1}} \\
-\frac{\Sigma_{\mathrm{g} 1 \mathrm{~g} 2}}{D_{2}} & \frac{\Sigma_{\mathrm{a} 2}}{D_{2}}
\end{array}\right) .
$$

The goal of this method is to obtain quantity $\xi_{\mathrm{g}}$, from which neutron flux can be obtained by using inverse matrix $\mathbb{R}$ in both energy groups. General solution of wave equation 5 is:

$$
\begin{aligned}
\xi_{\mathrm{g}}(\vec{r}, \phi) & =\int A_{\mathrm{g}}(\alpha) c n\left(\sqrt{\left|\lambda_{\mathrm{g}}\right|} \vec{r} \cos (\phi-\alpha)\right) d \alpha \\
& +\int B_{\mathrm{g}}(\alpha) \operatorname{sn}\left(\sqrt{\left|\lambda_{\mathrm{g}}\right|} \vec{r} \cos (\phi-\alpha)\right) d \alpha
\end{aligned}
$$

where $A_{\mathrm{g}}(\alpha)$ and $B_{\mathrm{g}}(\alpha)$ are constants dependent on interface of fuel assembly and function $s n$ and $c n$ are defined according to value of eigenvalue $\lambda_{\mathrm{g}}$, see table 1 .

$\begin{array}{ccc}\text { General function } & \text { Real function } & \text { Condition } \\ \operatorname{sn}(x) & \sin (x) & \lambda_{\mathrm{g}}<0 \\ & \sinh (x) & \lambda_{\mathrm{g}}>0 \\ \operatorname{cn}(x) & \cos (x) & \lambda_{\mathrm{g}}<0 \\ & \cosh (x) & \lambda_{\mathrm{g}}>0\end{array}$

TABLE 1. Function $s n$ and $c n$ for smooth neutron flux profile reconstruction

Given that, the interfaces of the fuel assembly are on discrete coordinates and discrete angles. The equation 8 can be rewritten as:

$$
\begin{aligned}
\xi_{\mathrm{g}}(\vec{r}, \phi) & =\sum_{i=0}^{2} A_{\mathrm{g}, i} c n\left(\sqrt{\left|\lambda_{\mathrm{g}}\right|} \vec{r} \cos \left(\phi-i \frac{\pi}{3}\right)\right) \\
& +\sum_{i=0}^{2} B_{\mathrm{g}, i} \operatorname{sn}\left(\sqrt{\left|\lambda_{\mathrm{g}}\right|} \vec{r} \cos \left(\phi-i \frac{\pi}{3}\right)\right),
\end{aligned}
$$

where constants $A_{\mathrm{g}, i}$ and $B_{\mathrm{g}, i}$ are determined by known mean value of neutron flux on each interface of fuel assembly. By express of mean value of quantity $\xi_{g}$ from equation 9, it could be obtained for each fuel assembly interface set of equation for $k=1,2, \ldots, 6$ as:

$$
\overline{\xi_{\mathrm{g}}(k)}=\sum_{i=0}^{2} A_{\mathrm{g}, i} a_{i, k}+\sum_{i=0}^{2} B_{\mathrm{g}, i} b_{i, k},
$$

where $a_{i, k}$ and $b_{i, k}$ are average values of chosen function over fuel assembly interface. The equation system can be solved due to known values $\overline{\xi_{\mathrm{g}}(k)}$ on fuel assembly interface, from which can be obtained constants $A_{\mathrm{g}, i}$ and $B_{\mathrm{g}, i}$. Based on known constants, course of quantity $\xi_{\mathrm{g}}(\vec{r}, \phi)$ can be determined in all fuel assemblies. Thanks to the knowledge $\xi_{\mathrm{g}}(\vec{r}, \phi)$, equation 6 can be solved. It is possible to calculate neutron flux in elementary volume of coordinates $\vec{r}$ and $\phi$ from the solution of equation 6 , 5

For more accurate reconstruction of neutron flux, there are included values of neutron flux in assembly corners. Equation system 9 is replaced by:

$$
\begin{aligned}
\xi_{\mathrm{g}}(\vec{r}, \phi) & =\sum_{i=0}^{5} A_{\mathrm{g}, i} c n\left(\sqrt{\left|\lambda_{\mathrm{g}}\right|} \vec{r} \cos \left(\phi-i \frac{\pi}{6}\right)\right) \\
& +\sum_{i=0}^{5} B_{\mathrm{g}, i} \operatorname{sn}\left(\sqrt{\left|\lambda_{\mathrm{g}}\right|} \vec{r} \cos \left(\phi-i \frac{\pi}{6}\right)\right) .
\end{aligned}
$$

\subsection{Reconstructed NEUtron FLuX APPLICATION TO MACROSCOPIC CROSS SECTION VALUES}

The rehomogenization is data preparation process based on more accurate distribution of neutron flux in fuel assembly. The data preparation process is based on the Flux-Weighted Volume method (FWV):

$$
\Sigma_{\mathrm{g}}=\frac{\sum_{h \in \mathrm{g}} \sum_{i} V_{i} \Sigma_{i, h} \Phi_{i, h}}{\sum_{h \in \mathrm{g}} \sum_{i} V_{i} \Phi_{i, h}},
$$

where $i$ indicates spatial discrete cells with different materials, $V_{i}$ are volumes of these cells and $\Sigma_{i, h}$ are macroscopic cross sections of cell $i$ and energy $h$, which is from interval $\mathrm{g}=\left(E_{\mathrm{g}-1}, E_{\mathrm{g}}\right)$ and $\Phi_{i, h}$ is neutron flux in cell $i$ and energy $h$.

It has been proven that the identically assembly data as the data homogenized from macroscopic cross sections for each cell can be prepared by FWV.

Using the reconstructed neutron flux and cell macroscopic cross sections from infinite lattice, the new data for fuel assemblies were obtained thank to equation 12.

\section{Method testing}

The method testing was performed based on data comparison with reference model case calculated with accurate deterministic code Helios. These cases could be divided as:

- fuel cases,

- cases with strong absorber,

- cases with regulation rods,

- cases with reflector assembly.

Test tasks are divided to two independent categories. The method was firstly tested using comparison with accurate data for neutron flux distribution calculation in fuel assembly - Test of the method. These data were found by simulating reference case in the microcode Helios. In the second step, the neutron flux distribution was found using data provided by diffusion approach - Practice test.

The flowchart of the data flow is in figure 3 The orange arrows show the data flow during the Test of the method. The green arrows show the data flow during the Practice test. The data from flux interpolation test had high deviation and due to it, they were not used for the rehomogenization process.

If the rehomogenized data are in better agreement with reference data and their change compared to the solution of infinite lattice in absolute value is more 


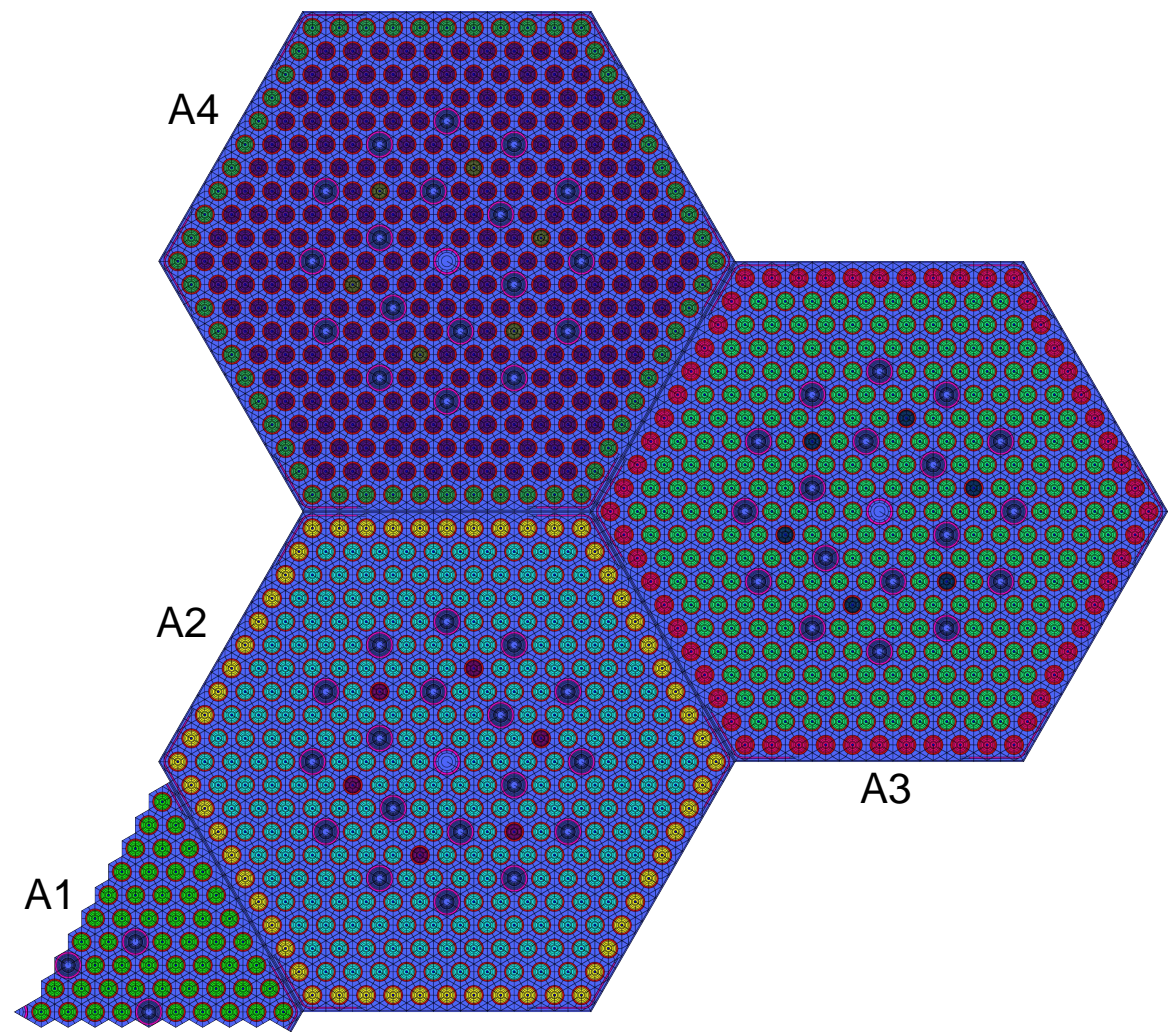

FiguRE 2. Illustration of larger case with caption of each fuel assembly.

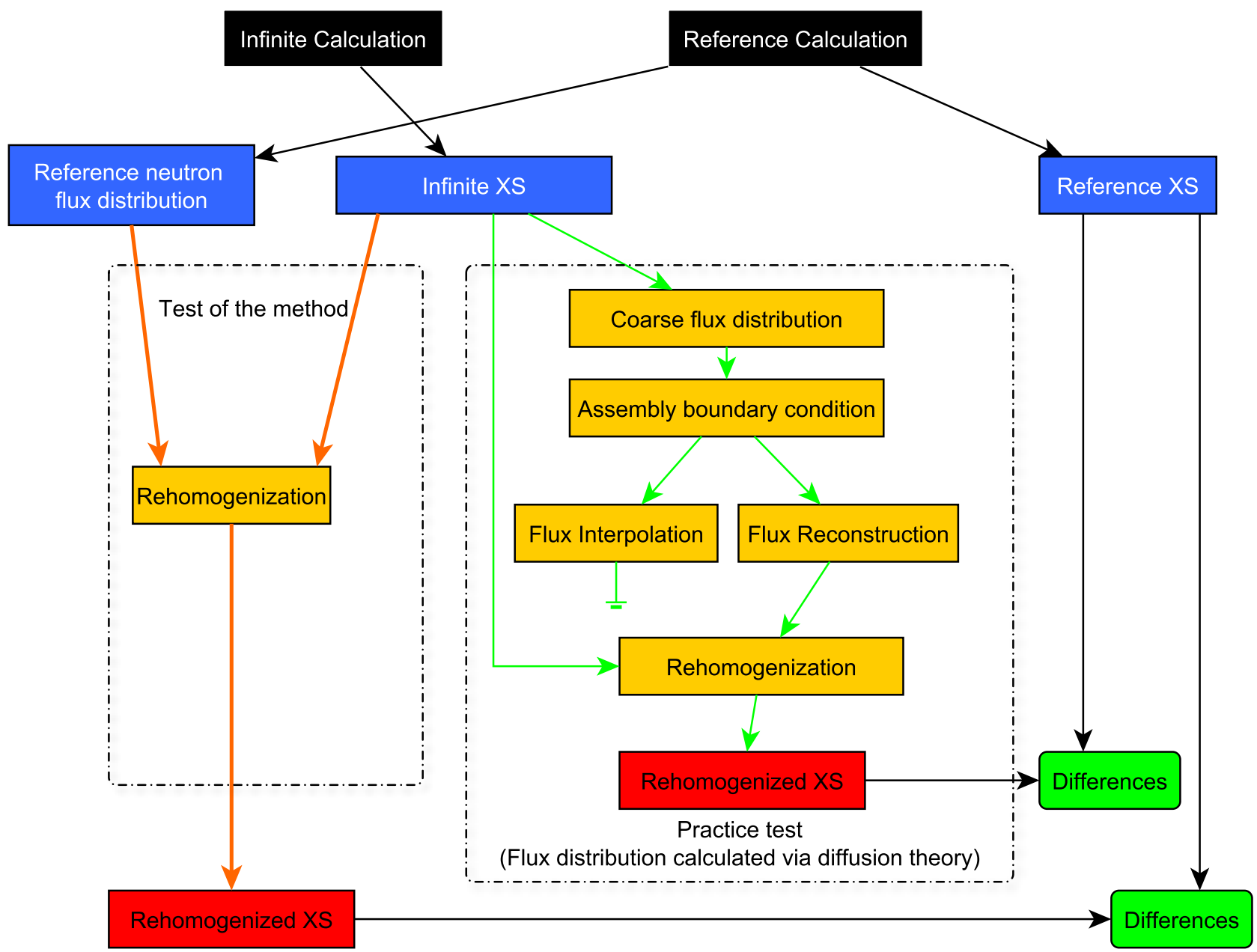

Figure 3. The flowchart of the both testing method. 


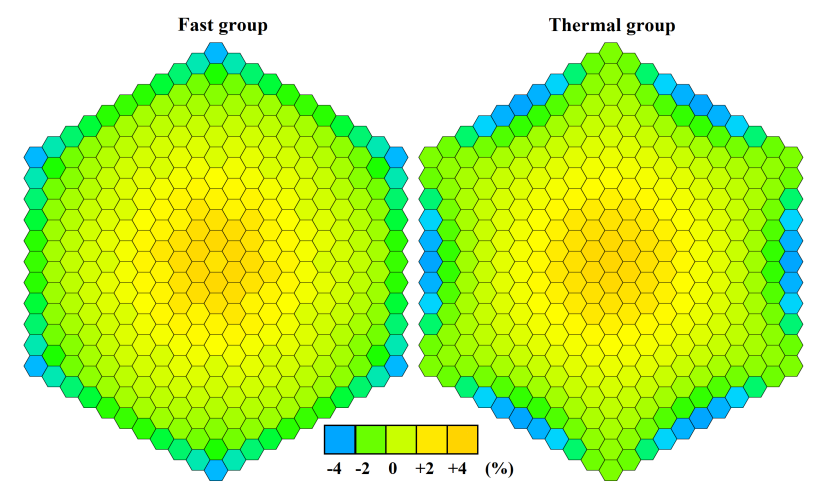

FiguRE 4. Deviations of smooth profile neutron flux reconstructions for A1 from accurate solution with using Andrea reconstruction process in case A with data from Helios.

than $10 \%$, than the data are with green colour. If the rehomogenized data are in wore agreement with reference data and their change compared to the solution of infinite lattice in absolute value is more than $10 \%$, than the data are with red colour. The results with lower absolute change than $10 \%$ are with black colour.

\subsection{TEST OF METHOD}

This part of testing is focused on analysis of all the benefits of the rehomogenization method in case of accurate input parameters for calculation of smooth neutron flux profile inside fuel assembly.

The monitored parameters were the neutron flux distribution and the macroscopic cross section. Moreover the eigenvalue was calculated from macroscopic cross section using two groups diffusion equation in infinite lattice.

\subsubsection{CASE A}

The case A consists of fuel assembly A1-a13A, A2a40A6 6. Fuel assembly a13A is situated in the middle of the case, around its are 6 fuel assemblies a40A6. The whole structure is simulated inside infinite lattice. It is quite realistic model of fuel assemblies distribution in a reactor core. Results of deviation of smooth profile neutron flux reconstruction with accurate Helios data for each fuel assembly and energy group are in figures 4 and 5

Figures 4 and 5 show good compliance of smooth profile neutron flux, obtained by reconstruction process, with reference calculation via Helios. The macroscopic cross sections prepared using reference calculation in case together with deviation of infinite lattice calculation, respectively rehomogenized solution from values prepared in case are in the table 2 Deviations were calculated using equation:

$$
\Delta_{\mathrm{ref}, \mathrm{x}}=\frac{\Sigma_{\mathrm{ref}}-\Sigma_{\mathrm{x}}}{\Sigma_{\mathrm{ref}}} \cdot 100,
$$

where quantity $\mathrm{x}$ means reconstructed macroscopic

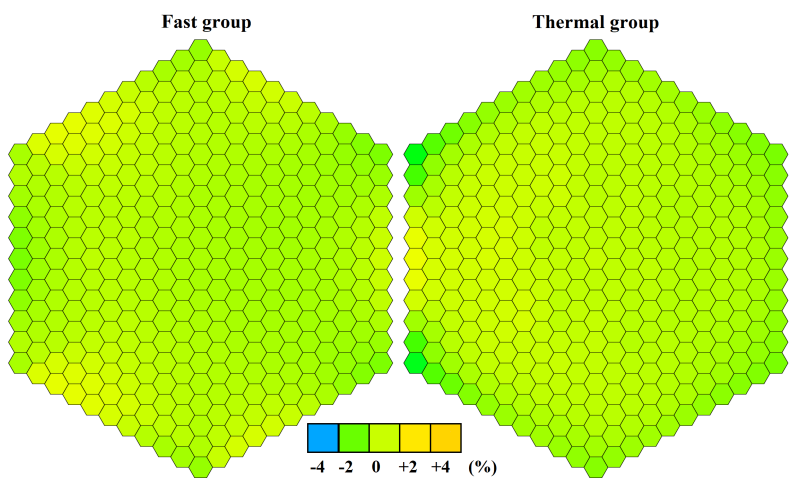

FiguRE 5. Deviations of smooth profile neutron flux reconstructions for A2 from accurate solution with using Andrea reconstruction process in case A with data from Helios.

\begin{tabular}{cccc}
\hline Type & $\Sigma_{\text {ref }}\left(\mathrm{cm}^{-1}\right)$ & $\Delta_{\text {ref, },}(\%)$ & $\Delta_{\text {ref,rec }}(\%)$ \\
\hline \multicolumn{4}{c}{ Fuel assembly A1 - a13A } \\
\hline$\Sigma_{\mathrm{a}, \mathrm{g} 1}$ & $8.07 \mathrm{E}-03$ & -0.748 & -0.580 \\
$\Sigma_{\mathrm{a}, \mathrm{g} 2}$ & $5.64 \mathrm{E}-02$ & -0.759 & -1.113 \\
$D_{\mathrm{g} 1}$ & $1.32 \mathrm{E}+00$ & -2.445 & -2.454 \\
$D_{\mathrm{g} 2}$ & $4.09 \mathrm{E}-01$ & 4.562 & 4.410 \\
$\nu \Sigma_{\mathrm{f}, \mathrm{g} 1}$ & $3.95 \mathrm{E}-03$ & -1.684 & -1.482 \\
$\nu \Sigma_{\mathrm{f}, \mathrm{g} 2}$ & $6.13 \mathrm{E}-02$ & -0.640 & -1.287 \\
$\kappa \Sigma_{\mathrm{f}, \mathrm{g} 1}$ & $4.99 \mathrm{E}-14$ & -1.607 & -1.405 \\
$\kappa \Sigma_{\mathrm{f}, \mathrm{g} 2}$ & $8.15 \mathrm{E}-13$ & -0.640 & -1.286 \\
$\Sigma_{\mathrm{g} 1 \mathrm{~g} 2}$ & $1.86 \mathrm{E}-02$ & -2.448 & -2.570 \\
$\Sigma_{\mathrm{g} 2 \mathrm{~g} 1}$ & $1.01 \mathrm{E}-03$ & 9.454 & 9.505 \\
\hline \multicolumn{4}{c}{ Fuel assembly A2-a40A6 } \\
\hline$\Sigma_{\mathrm{a}, \mathrm{g} 1}$ & $9.74 \mathrm{E}-03$ & 0.097 & -0.117 \\
$\Sigma_{\mathrm{a}, \mathrm{g} 2}$ & $1.07 \mathrm{E}-01$ & 0.106 & -0.088 \\
$D_{\mathrm{g} 1}$ & $1.34 \mathrm{E}+00$ & 0.285 & 0.301 \\
$D_{\mathrm{g} 2}$ & $3.99 \mathrm{E}-01$ & -1.117 & -1.127 \\
$\nu \Sigma_{\mathrm{f}, \mathrm{g} 1}$ & $7.43 \mathrm{E}-03$ & 0.128 & -0.103 \\
$\nu \Sigma_{\mathrm{f}, \mathrm{g} 2}$ & $1.60 \mathrm{E}-01$ & 0.132 & -0.125 \\
$\kappa \Sigma_{\mathrm{f}, \mathrm{g} 1}$ & $9.59 \mathrm{E}-14$ & 0.125 & -0.107 \\
$\kappa \Sigma_{\mathrm{f}, \mathrm{g} 2}$ & $2.12 \mathrm{E}-12$ & 0.132 & -0.125 \\
$\Sigma_{\mathrm{g} 1 \mathrm{~g} 2}$ & $1.64 \mathrm{E}-02$ & 0.231 & 0.320 \\
$\Sigma_{\mathrm{g} 2 \mathrm{~g} 1}$ & $1.64 \mathrm{E}-03$ & -1.144 & -1.136 \\
\hline
\end{tabular}

TABlE 2. Macroscopic cross sections prepared using simulation in reference case (ref) for case A and deviations solution in infinite lattice, respectively rehomogenized cross sections.

cross sections or cross sections prepared in infinite lattice calculation.

Besides macroscopic cross sections, eigenvalues calculated from two group diffusion equation and rehomogenized data were analysed. The values are provided in table 3 Eigenvalue provides additional integral quantity, decisive about suitability of rehomogenization method of macroscopic cross sections. 


\begin{tabular}{cccc}
\hline FA & $k_{\text {ref }}^{\infty}$ & $\Delta k_{\text {ref, } \infty}^{\infty}$ & $\Delta k_{\text {ref,rec }}^{\infty}$ \\
\hline A1 & 0.903885 & $-282 \mathrm{pcm}$ & $-533 \mathrm{pcm}$ \\
$\mathrm{A} 2$ & 1.218033 & $59 \mathrm{pcm}$ & $47 \mathrm{pcm}$ \\
\hline
\end{tabular}

TABLE 3. Eigenvalues calculated from macroscopic cross section of case A using two group diffusion equation.

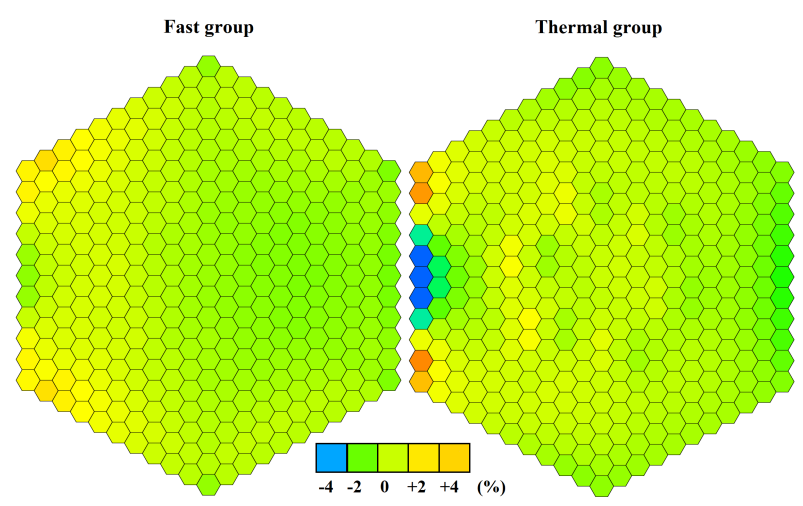

Figure 6. Deviations of smooth profile neutron flux reconstructions for A2 from accurate solution with using Andrea reconstruction process in case $\mathrm{F}$ with data from Helios.

\subsubsection{CASE F}

Case $\mathrm{F}$ is larger case, in which was central fuel assembly replaced by model of HRK regulation assembly. Model with HRK was chosen as a real problem with higher neutron absorption than in model with regulation cluster. Regulation by HRK assembly is used for instance in reactor WWER-440, where is fuel assembly replaced by absorbing part. Interlacing of reconstructed neutron flux for fuel assembly adjoining to HRK assembly (A2) is in figure 6 The neutron flux deviation are on the level of -5 to $3 \%$ in case of interface next to the HRK assembly. The neutron flux was reconstructed for other fuel assemblies with deviations around $1 \%$ in comparison with accurate solution from Helios.

\subsubsection{CASE G}

Within the complete description of possible use of rehomogenization method, there was also simulated situation with reflector assembly. Central assembly was replaced by part of WWER-1000 reflector assembly, the rest three fuel assemblies were a40A6. The figure 7 shows comparison of reconstructed neutron flux with accurate solution obtained by simulation in Helios for fuel assembly next to the reflector assembly. Maximal deviations in the reconstruction are in the thermal group near the reflector interface. The deviations were up to $7 \%$.

\subsection{Practice test}

In the second step there were used boundary conditions calculated via Andrea macrocode for neutron flux distribution calculation. Under this subsection

\begin{tabular}{cccc}
\hline Type & $\Sigma_{\text {ref }}\left(\mathrm{cm}^{-1}\right)$ & $\Delta_{\text {ref, } \infty}(\%)$ & $\Delta_{\text {ref,rec }}(\%)$ \\
\hline$\Sigma_{\mathrm{a}, \mathrm{g} 1}$ & $9.74 \mathrm{E}-03$ & 0.119 & -0.088 \\
$\Sigma_{\mathrm{a}, \mathrm{g} 2}$ & $1.07 \mathrm{E}-01$ & -0.257 & -0.572 \\
$D_{\mathrm{g} 1}$ & $1.34 \mathrm{E}+00$ & 0.418 & 0.433 \\
$D_{\mathrm{g} 2}$ & $4.05 \mathrm{E}-01$ & 0.372 & 0.339 \\
$\nu \Sigma_{\mathrm{f}, \mathrm{g} 1}$ & $7.43 \mathrm{E}-03$ & 0.116 & -0.112 \\
$\nu \Sigma_{\mathrm{f}, \mathrm{g} 2}$ & $1.59 \mathrm{E}-01$ & -0.089 & -0.376 \\
$\kappa \Sigma_{\mathrm{f}, \mathrm{g} 1}$ & $9.59 \mathrm{E}-14$ & 0.119 & -0.110 \\
$\kappa \Sigma_{\mathrm{f}, \mathrm{g} 2}$ & $2.12 \mathrm{E}-12$ & -0.089 & -0.376 \\
$\Sigma_{\mathrm{s}, \mathrm{g} 1}$ & $1.64 \mathrm{E}-02$ & -0.146 & -0.059 \\
$\Sigma_{\mathrm{s}, \mathrm{g} 2}$ & $1.67 \mathrm{E}-03$ & 0.400 & 0.351 \\
\hline
\end{tabular}

TABLE 4. Macroscopic cross sections prepared using simulation in reference case (ref) for case $\mathrm{F}$ and deviations solution in infinite lattice, respectively rehomogenized cross sections.

\begin{tabular}{ccc}
\hline$k_{\mathrm{ref}}^{\infty}$ & $\Delta k_{\mathrm{ref}, \infty}^{\infty}$ & $\Delta k_{\mathrm{ref}, \mathrm{rec}}^{\infty}$ \\
\hline 1.218522 & $108 \mathrm{pcm}$ & $177 \mathrm{pcm}$ \\
\hline
\end{tabular}

TABLE 5. Eigenvalues calculated from macroscopic cross section of case $\mathrm{F}$ using two group diffusion equation.

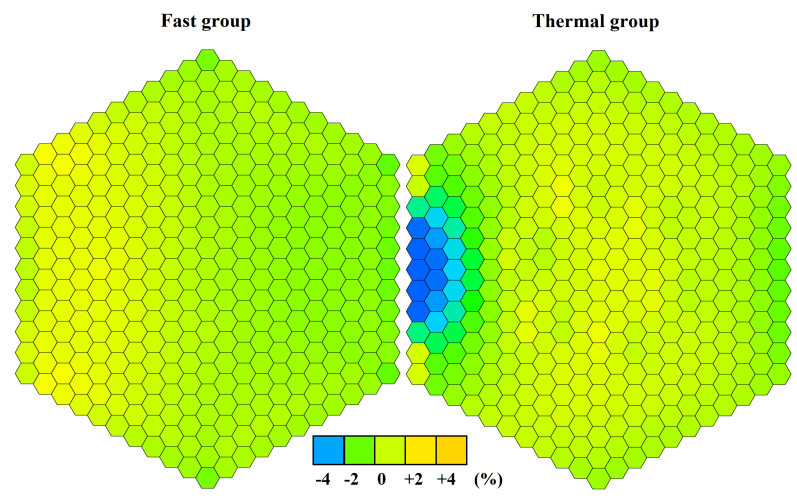

FiguRE 7. Deviations of smooth profile neutron flux reconstructions for A2 from accurate solution with using Andrea reconstruction process in case $\mathrm{G}$ with data from Helios.

\begin{tabular}{cccc}
\hline Type & $\Sigma_{\text {ref }}\left(\mathrm{cm}^{-1}\right)$ & $\Delta_{\text {ref, } \infty}(\%)$ & $\Delta_{\text {ref,rec }}(\%)$ \\
\hline$\Sigma_{\mathrm{a}, \mathrm{g} 1}$ & $9.78 \mathrm{E}-03$ & 0.540 & 0.335 \\
$\Sigma_{\mathrm{a}, \mathrm{g} 2}$ & $1.07 \mathrm{E}-01$ & -0.005 & -0.325 \\
$D_{\mathrm{g} 1}$ & $1.34 \mathrm{E}+00$ & 0.256 & 0.271 \\
$D_{\mathrm{g} 2}$ & $4.03 \mathrm{E}-01$ & -0.210 & -0.245 \\
$\nu \Sigma_{\mathrm{f}, \mathrm{g} 1}$ & $7.44 \mathrm{E}-03$ & 0.196 & -0.032 \\
$\nu \Sigma_{\mathrm{f}, \mathrm{g} 2}$ & $1.59 \mathrm{E}-01$ & -0.038 & -0.328 \\
$\kappa \Sigma_{\mathrm{f}, \mathrm{g} 1}$ & $9.60 \mathrm{E}-14$ & 0.238 & 0.010 \\
$\kappa \Sigma_{\mathrm{f}, \mathrm{g} 2}$ & $2.12 \mathrm{E}-12$ & -0.038 & -0.328 \\
$\Sigma_{\mathrm{s}, \mathrm{g} 1}$ & $1.65 \mathrm{E}-02$ & 0.702 & 0.787 \\
$\Sigma_{\mathrm{s}, \mathrm{g} 2}$ & $1.67 \mathrm{E}-03$ & 0.939 & 0.887 \\
\hline
\end{tabular}

TABlE 6. Macroscopic cross sections prepared using simulation in reference case (ref) for case $\mathrm{G}$ and deviations solution in infinite lattice, respectively rehomogenized cross sections. 


\begin{tabular}{ccc}
\hline$k_{\text {ref }}^{\infty}$ & $\Delta k_{\text {ref, } \infty}^{\infty}$ & $\Delta k_{\text {ref,rec }}^{\infty}$ \\
\hline 1.216401 & $-104 \mathrm{pcm}$ & $-33 \mathrm{pcm}$ \\
\hline
\end{tabular}

TABLE 7. Eigenvalues calculated from macroscopic cross section of case $\mathrm{G}$ using two group diffusion equation.

\begin{tabular}{cccc}
\hline Type & $\Sigma_{\text {ref }}\left(\mathrm{cm}^{-1}\right)$ & $\Delta_{\text {ref }, \infty}(\%)$ & $\Delta_{\text {ref, rec }}(\%)$ \\
\hline$\Sigma_{\mathrm{a}, \mathrm{g} 1}$ & $9.74 \mathrm{E}-03$ & 2.500 & 2.507 \\
$\Sigma_{\mathrm{a}, \mathrm{g} 2}$ & $1.07 \mathrm{E}-01$ & 10.100 & 10.171 \\
$\Sigma_{\mathrm{f}, \mathrm{g} 1}$ & $2.93 \mathrm{E}-03$ & 0.127 & 0.127 \\
$\Sigma_{\mathrm{f}, \mathrm{g} 2}$ & $6.56 \mathrm{E}-02$ & 0.131 & 0.134 \\
$\Sigma_{\mathrm{s}, \mathrm{g} 1 \mathrm{~g} 1}$ & $5.23 \mathrm{E}-01$ & -0.018 & -0.018 \\
$\Sigma_{\mathrm{s}, \mathrm{g} 1 \mathrm{~g} 2}$ & $1.64 \mathrm{E}-02$ & 0.232 & 0.232 \\
$\Sigma_{\mathrm{s}, \mathrm{g} 2 \mathrm{~g} 1}$ & $1.64 \mathrm{E}-03$ & -1.140 & -1.143 \\
$\Sigma_{\mathrm{s}, \mathrm{g} 2 \mathrm{~g} 2}$ & $1.25 \mathrm{E}+00$ & 0.111 & 0.112 \\
$D_{\mathrm{g} 1}$ & $1.34 \mathrm{E}+00$ & 0.285 & 0.285 \\
$D_{\mathrm{g} 2}$ & $3.99 \mathrm{E}-01$ & -1.120 & -1.117 \\
\hline
\end{tabular}

TABle 8. $\Sigma$ homogenized in reference case (ref) and deviation infinite and reconstructed cross sections for case A with boundary conditions from Andrea.

the neutron fluxes on the interfaces of fuel assemblies, macroscopic cross sections and in conclusion eigenvalues are compared at first.

The eigenvalues in this approach were calculated via macrocode Andrea. The neutron leakage from the system was calculated using the Helios microcode (using diffusion coefficient and buckling factor). This neutron leakage from the system was installed to macrocode Andrea. The eigenvalue could be reached as $k_{\text {eff }}=1$ with data from case model. The deviation of eigenvalue from 1 is given by deviations in macroscopic cross sections from reference solution. The deviation of eigenvalue from criticality is in this case integral parameter of rehomogenization.

\section{CAse A}

The macroscopic cross sections for fuel assembly A2 in case $\mathrm{A}$ are compared in the table 8 .

The deviation of eigenvalue from criticality increased from $20 \mathrm{pcm}$ to $30 \mathrm{pcm}$ in this configuration with using rehomogenized cross section.

\section{CAse F}

The compared macroscopic cross sections for fuel assembly A2 in case $\mathrm{F}$ are in the table 9

The deviation of eigenvalue from criticality decreased from $314 \mathrm{pcm}$ to $123 \mathrm{pcm}$ in this configuration with using rehomogenized cross section.

\section{CAse G}

The macroscopic cross sections for fuel assembly A2 in case $\mathrm{G}$ are compared in the table 10

The deviation of eigenvalue from criticality decreased from $99 \mathrm{pcm}$ to $46 \mathrm{pcm}$ in this configuration with using rehomogenized cross section.

\begin{tabular}{cccc}
\hline Type & $\Sigma_{\text {ref }}\left(\mathrm{cm}^{-1}\right)$ & $\Delta_{\text {ref }, \infty}(\%)$ & $\Delta_{\text {ref, rec }}(\%)$ \\
\hline$\Sigma_{\mathrm{a}, \mathrm{g} 1}$ & $9.74 \mathrm{E}-03$ & 2.497 & 2.507 \\
$\Sigma_{\mathrm{a}, \mathrm{g} 2}$ & $1.07 \mathrm{E}-01$ & 10.104 & 10.171 \\
$\Sigma_{\mathrm{f}, \mathrm{g} 1}$ & $2.93 \mathrm{E}-03$ & 0.127 & 0.127 \\
$\Sigma_{\mathrm{f}, \mathrm{g} 2}$ & $6.56 \mathrm{E}-02$ & 0.131 & 0.134 \\
$\Sigma_{\mathrm{s}, \mathrm{g} 1 \mathrm{~g} 1}$ & $5.23 \mathrm{E}-01$ & -0.018 & -0.018 \\
$\Sigma_{\mathrm{s}, \mathrm{g} 1 \mathrm{~g} 2}$ & $1.64 \mathrm{E}-02$ & 0.232 & 0.232 \\
$\Sigma_{\mathrm{s}, \mathrm{g} 2 \mathrm{~g} 1}$ & $1.64 \mathrm{E}-03$ & -1.143 & -1.143 \\
$\Sigma_{\mathrm{s}, \mathrm{g} 2 \mathrm{~g} 2}$ & $1.25 \mathrm{E}+00$ & 0.111 & 0.112 \\
$D_{\mathrm{g} 1}$ & $1.34 \mathrm{E}+00$ & 0.285 & 0.285 \\
$D_{\mathrm{g} 2}$ & $3.99 \mathrm{E}-01$ & -1.116 & -1.117 \\
\hline
\end{tabular}

TABLE 9. $\Sigma$ homogenized in reference case (ref) and deviation infinite and reconstructed cross sections for case $\mathrm{F}$ with boundary conditions from Andrea.

\begin{tabular}{cccc}
\hline Type & $\Sigma_{\text {ref }}\left(\mathrm{cm}^{-1}\right)$ & $\Delta_{\text {ref }, \infty}(\%)$ & $\Delta_{\text {ref, rec }}(\%)$ \\
\hline$\Sigma_{\mathrm{a}, \mathrm{g} 1}$ & $9.77 \mathrm{E}-03$ & 2.783 & 2.794 \\
$\Sigma_{\mathrm{a}, \mathrm{g} 2}$ & $1.07 \mathrm{E}-01$ & 9.725 & 9.789 \\
$\Sigma_{\mathrm{f}, \mathrm{g} 1}$ & $2.93 \mathrm{E}-03$ & 0.141 & 0.141 \\
$\Sigma_{\mathrm{f}, \mathrm{g} 2}$ & $6.54 \mathrm{E}-02$ & -0.126 & -0.123 \\
$\Sigma_{\mathrm{s}, \mathrm{g} 1 \mathrm{~g} 1}$ & $5.23 \mathrm{E}-01$ & 0.091 & 0.091 \\
$\Sigma_{\mathrm{s}, \mathrm{g} 1 \mathrm{~g} 2}$ & $1.65 \mathrm{E}-02$ & 0.475 & 0.475 \\
$\Sigma_{\mathrm{s}, \mathrm{g} 2 \mathrm{~g} 1}$ & $1.67 \mathrm{E}-03$ & 0.678 & 0.678 \\
$\Sigma_{\mathrm{s}, \mathrm{g} 2 \mathrm{~g} 2}$ & $1.25 \mathrm{E}+00$ & -0.343 & -0.342 \\
$D_{\mathrm{g} 1}$ & $1.34 \mathrm{E}+00$ & 0.371 & 0.371 \\
$D_{\mathrm{g} 2}$ & $4.03 \mathrm{E}-01$ & -0.081 & -0.081 \\
\hline
\end{tabular}

TABLE 10. $\Sigma$ homogenized in reference case (ref) and deviation infinite and reconstructed cross sections for case $\mathrm{G}$ with boundary conditions from Andrea.

\subsection{Results eVAluation}

The benefits from rehomogenization process with the reference neutron flux are not too noticeable and macroscopic cross sections change a little. This fact was found during the analysis based on the accurate data from the transport code Helios. Total influence of rehomogenization process showed up beneficial in case A case G.

The macroscopic cross section for all fuel assemblies were apparently much more dependent on the neutron spectra during the data preparation process, than on the distribution of neutron flux. Unfortunately the spectral homogenization process could not be solved without knowledge of loading pattern.

Deviations of neutron flux on the interfaces of fuel assembly between accurate solution from transport code and diffusion approach are up to $15 \%$. The macroscopic cross sections report the same behaviour as in the case of the accurate boundary values despite such deviations in boundary conditions for reconstruction.

Oppositely to the Test of Method task, there were registered benefits in case of fuel assembly next to the HRK assembly and next to the reflector assembly, but no benefits in case consisted from two fuel assemblies. The neutron flux tilt is apparently too low to 
bring better results because too high deviations in the boundary neutron fluxes and due to there is no way to prepare better macroscopic data for these problem.

\section{Conclusion}

The main goal of study is to analyse the benefits, which the method can bring to the full core calculation. New sets of macroscopic data were calculated via FWV with reconstructed neutron flux and macroscopic cross sections (calculated in infinite lattice) for each cell. The whole method was tested with input data for reconstruction process obtained by simulation in Helios. The general trend of the rehomogenized data was not found and due to there is no way to find total influence on the calculation. Results of eigenvalue show that rehomogenization can bring better results in case $A$ and case $G$.

In the second step, the method was tested with neutron flux boundary condition calculated by diffusion code Andrea. Benefits of this approach were minimal, because the cross sections were still the same, but total benefit was positive. Eigenvalues with rehomogenized macroscopic cross section were relatively identical to eigenvalue from reference case.

During this study was found that neutron flux inside fuel assemblies can not be approximated by easy polynomial functions, but there is need to use reconstruction method based on a solving diffusion equation. Despite the correctly reconstructed neutron flux shape, the macroscopic cross sections do not change significantly and this change was not essential to calculation via Andrea. This situation led to the question if is possible to separate spatial and spectral homogenization process. Probably this effect can be analysed by performing rehomogenization process using more neutron groups.

\section{LIST OF SYMBOLS}

$\Phi(x, y)$ Neutron flux in the cell with coordinates $x$ and $y$ $\left[\mathrm{cm}^{-2} \mathrm{~s}^{-1}\right]$

$\bar{\Phi}$ Mean value of neutron flux in fuel assembly $\left[\mathrm{cm}^{-2} \mathrm{~s}^{-1}\right]$

$\vec{\Phi} \quad$ Vector of grouped scalar neutron fluxes $\left[\mathrm{cm}^{-2} \mathrm{~s}^{-1}\right]$
$\Sigma_{i \mathrm{~g}} \quad$ Macroscopic cross section of reaction $i$ and energy group g $\left[\mathrm{cm}^{-1}\right]$

$\nu \quad$ Neutron fission yield $[-]$

$\kappa \quad$ Energy released during fission process $[-]$

$k_{\text {eff }}$ Effective eigenvalue $[-]$

$D_{\mathrm{g}}$ Diffusion coefficient of group $\mathrm{g}[\mathrm{cm}]$

$V_{i} \quad$ Volume of cell $i\left[\mathrm{~cm}^{3}\right]$

$k^{\infty} \quad$ Eigenvalue calculated using two group diffusion equation $[-]$

$\Delta k_{\min , \mathrm{x}}^{\infty}$ Deviation of eigenvalue of calculation x calculated with two group diffusion equation from reference calculation $[\mathrm{pcm}]$

a Index for absorption [-]

f Index for fission [-]

g1g2 Index for scatter from group 1 to group 2 [-]

g2g1 Index for scatter from group 2 to group 1 [-]

min Index for reference calculation in case [-]

$\infty$ Index for calculation in infinite lattice [-]

rec Index for calculation from rehomogenized data $[-]$

\section{REFERENCES}

[1] M. A. Jessee, M. D. DeHart. Newt: A new transport algorithm for two-dimensional discreteordinates analysis in non-orthogonal geometries. ORNL/TM-2005/39, 2016.

[2] C. Wemple, H.-N. Gheorghiu, R. Stamm'ler, E. Villarino. The helios-2 lattice physics code. https://www.studsvik.com/SharepointFiles/The\% 20HELIOS- $2 \%$ 20Lattice $\% 20$ Physics $\% 20$ Code.pdf.

[3] J. Leppänen. Serpent - a Continous-energy Monte Carlo Reactor Physics Burnup Calculation Code. VTT Technical Research Centre of Finland, 2015.

[4] A. Dall'Osso. A spatial rehomogenization method in nodal calculations. Science direct, 2006. https://www.researchgate.net/publication/ 245135037_A_spatial_rehomogenization_method_in_ nodal_calculations

[5] F. Havlůj, R. Vočka. ANDREA developer documentation. Nuclear Research Institute at Řež, 2005-2013.

[6] F. Havlůj, J. Hejzlar, R. Vočka. ANDREA data libraries, VVER-1000/TVSA-T. Nuclear Research Institute at Řě̌, 2016-. 